\title{
The integrated stress response prevents demyelination by protecting oligodendrocytes against immune-mediated damage
}

\author{
Wensheng Lin, ${ }^{1}$ Samantha L. Bailey, ${ }^{2}$ Hanson Ho, ${ }^{1}$ Heather P. Harding, ${ }^{3}$ David Ron, ${ }^{3}$ \\ Stephen D. Miller, ${ }^{2}$ and Brian Popko ${ }^{1}$ \\ 1Jack Miller Center for Peripheral Neuropathy, Department of Neurology, The University of Chicago, Chicago, Illinois, USA \\ 2Department of Microbiology-Immunology and Interdepartmental Immunobiology Center, Feinberg School of Medicine, Northwestern University, \\ Chicago, Illinois, USA. ${ }^{3}$ Skirball Institute of Biomolecular Medicine, New York University School of Medicine, New York, New York, USA.
}

\begin{abstract}
In response to ER stress, the pancreatic endoplasmic reticulum kinase (PERK) coordinates an adaptive program known as the integrated stress response (ISR) by phosphorylating the $\alpha$ subunit of eukaryotic translation initiation factor $2(\mathrm{eIF} 2 \alpha)$. IFN- $\gamma$, which activates the ER stress response in oligodendrocytes, is believed to play a critical role in the immune-mediated CNS disorder multiple sclerosis (MS) and its mouse model, experimental autoimmune encephalomyelitis (EAE). Here we report that CNS delivery of IFN- $\gamma$ before EAE onset ameliorated the disease course and prevented demyelination, axonal damage, and oligodendrocyte loss. The beneficial effects of IFN- $\gamma$ were accompanied by PERK activation in oligodendrocytes and were abrogated in PERK-deficient animals. Our results indicate that IFN- $\gamma$ activation of PERK in mature oligodendrocytes attenuates EAE severity and suggest that therapeutic approaches to activate the ISR could prove beneficial in MS.
\end{abstract}

\section{Introduction}

Phosphorylation of the $\alpha$ subunit of eukaryotic translation initiation factor $2(\mathrm{eIF} 2 \alpha)$ on serine 51 is a highly conserved point of convergence among distinct signaling pathways that adapt eukaryotic cells to diverse stressful conditions. eIF $2 \alpha$ phosphorylation promotes a stress-resistant state by global attenuation of protein biosynthesis and induction of numerous stress-induced cytoprotective genes. This eIF $2 \alpha$ phosphorylation-dependent, stress-inducible pathway has been referred to as the integrated stress response (ISR; refs. 1-3). Four different kinases are known to phosphorylate eIF2 $\alpha$. Pancreatic endoplasmic reticulum kinase (PERK), an ERlocalized eIF $2 \alpha$ kinase, is activated by ER stress, the stress of accumulating unfolded or misfolded proteins in the ER (1-3). General control nonderepressible-2 (GCN2) kinase is activated by amino acid starvation $(1,2)$. Double-stranded RNA-dependent protein kinase (PKR) and heme-regulated inhibitor (HRI) are activated by viral infection and iron deficiency, respectively (2).

Demyelination and oligodendrocyte loss are hallmarks of the immune-mediated CNS disorder MS and its animal model, EAE $(4,5)$. The T cell-derived pleiotropic cytokine IFN- $\gamma$ is regarded as a major proinflammatory cytokine that promotes myelin damage in MS and $\operatorname{EAE}(6,7)$. Normal functions of IFN- $\gamma$ include the promotion of Th1 $\mathrm{T}$ cell differentiation, activation of microglia/ macrophages, induction of MHC class I and MHC class II expression, and upregulation of many inflammatory mediators such as TNF- $\alpha$ and iNOs. IFN- $\gamma$ is undetectable in the normal CNS but becomes measurable during CNS inflammation, such as in the symptomatic phase of MS and $\operatorname{EAE}(6,7)$. Administration of

Nonstandard abbreviations used: eIF2 $\alpha, \alpha$ subunit of eukaryotic translation initiation factor 2; GFAP, glial fibrillary acidic protein; ISR, integrated stress response; MBP, myelin basic protein; MOG, myelin oligodendrocyte glycoprotein; $\mathrm{p}-$, phosphorylated; PERK, pancreatic endoplasmic reticulum kinase; PID, postimmunization day; TRE, tetracycline response element; tTA, tetracycline-controlled transactivator.

Conflict of interest: The authors have declared that no conflict of interest exists. Citation for this article: J. Clin. Invest. 117:448-456 (2007). doi:10.1172/JCI29571.
IFN- $\gamma$ to MS patients and EAE mice enhances inflammation in the CNS and exacerbates clinical symptoms (8-10). Enforced expression of IFN- $\gamma$ in the CNS of transgenic mice also results in CNS inflammation and myelin abnormalities $(11,12)$. Nevertheless, evidence is accumulating that IFN- $\gamma$ is not exclusively detrimental in the pathogenesis of MS and $\operatorname{EAE}(13,14)$. Mice with a mutation in either the gene encoding IFN- $\gamma$ or that encoding its receptor remain susceptible to EAE and in fact develop EAE with higher morbidity and mortality $(15,16)$. Moreover, deletion of IFN- $\gamma$ or its receptor makes resistant mouse strains susceptible to EAE (17). Importantly, it has also been shown that IFN- $\gamma$ delivered intrathecally before disease onset protects C57BL/6 mice from EAE (18). Here we confirm the protective role of early CNS delivery of IFN- $\gamma$ using transgenic mice that allow for temporally regulated delivery of IFN- $\gamma$ to the CNS using a tetracycline controllable system (19-21) and demonstrate that these protective effects against EAE are mediated by activation of the PERK-phosphorylated eIF2 $\alpha$ $($ PERK-p-eIF $2 \alpha$ ) ISR pathway in oligodendrocytes. Our observations suggest that therapeutic approaches to activate the ISR could prove beneficial in MS.

\section{Results}

IFN- $\gamma$ protects against EAE-induced demyelination. To assess the role of IFN- $\gamma$ in the pathogenesis of EAE, we used transgenic mice that allow for temporally regulated delivery of IFN- $\gamma$ to the CNS using a tetracycline-controllable system (19-21). In these animals, expression of the tetracycline-controlled transactivator (tTA) is driven by the astrocyte-specific transcriptional regulatory region of the glial fibrillary acidic protein (GFAP) gene, and a mouse IFN- $\gamma$ expressing gene has been placed under the transcriptional control of the tetracycline response element (TRE). We refer to these mice as GFAP/tTA;TRE/Ifng. In this experimental system, 6-weekold tTA compound transgenic mice that had been maintained on doxycycline from conception (to suppress the expression of the TRE/Ifng transgene) were immunized with the myelin peptide 


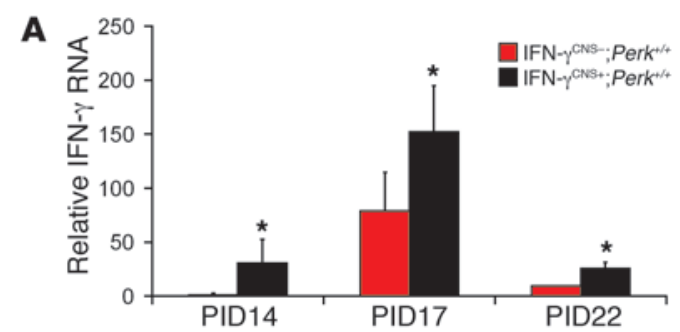

B

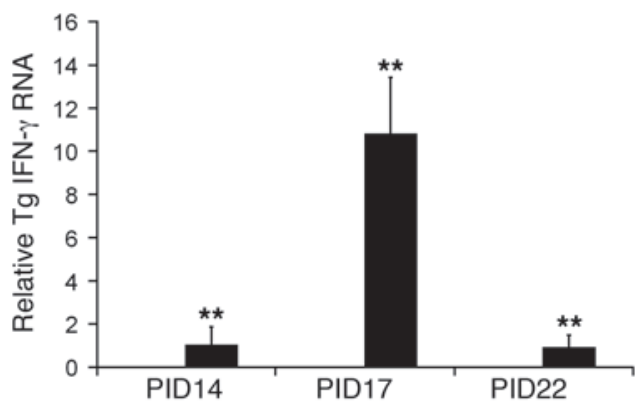

C

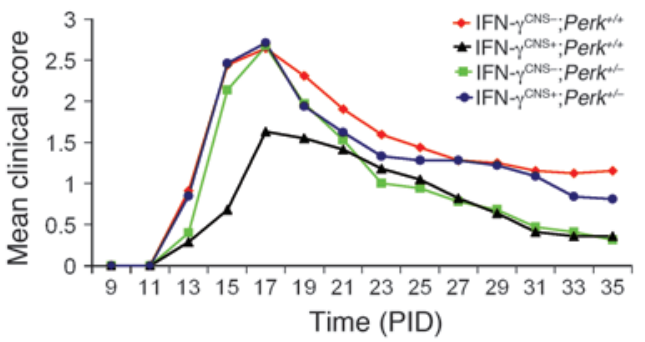

antigen myelin oligodendrocyte glycoprotein 35-55 (MOG $\left.{ }_{35-55}\right)$ in order to induce EAE. Doxycycline repression was discontinued in one group of compound transgenic mice on a Perk wild type background to induce CNS expression of IFN- $\gamma$ at the time of immunization and before EAE onset $\left(\right.$ IFN- $\gamma^{\mathrm{CNS}+} ;$ Perk $\left.^{+/+}\right)$. A second group of immunized, compound transgenic mice on a Perk wild type background was maintained under doxycycline repression to serve as controls (IFN- $\gamma^{\text {CNS-}} ;$ Perk $^{+/+}$).

Real-time PCR analysis showed that the levels of IFN- $\gamma$ mRNA in the spinal cords of control IFN- $\gamma^{\mathrm{CNS}-} ; \mathrm{Perk}^{+/+}$mice became detectable on postimmunization day 14 (PID14), the time of EAE onset, peaked with disease manifestations at PID17, and declined during the recovery stage, by PID22 (Figure 1A). This temporal profile is consistent with the published observations of Okuda et al. (22). The level of IFN- $\gamma$ mRNA in the spinal cords of IFN- $\gamma^{\mathrm{CNS}+} ;$ Perk $^{+/+}$ mice was significantly higher than in control IFN- $\gamma^{\mathrm{CNS}_{-}} ;$Perk $^{+/+}$mice at PID14, PID17, and PID22. Moreover, the IFN- $\gamma$ mRNA expressed by the TRE/Ifng transgene was detected exclusively in the CNS of IFN- $\gamma^{\mathrm{CNS}^{+}} ;$Perk $^{+/+}$mice at PID14 (Figure 1B), but not at earlier time points (data not shown). In addition, ELISA analysis showed that the level of IFN- $\gamma$ protein was markedly higher in the spinal cords of IFN- $\gamma^{\mathrm{CNS}+}$;Perk ${ }^{+/+}$mice (approximately 500 $\mathrm{pg} / \mathrm{mg}$ ) at PID17, the peak of disease, compared with control IFN- $\gamma^{\mathrm{CNS}-} ;$ Perk $^{+/+}$mice (approximately $60 \mathrm{pg} / \mathrm{mg}$ ). The time of disease onset was similar in the IFN- $\gamma^{\mathrm{CNS}+} ; \mathrm{Perk}^{+/+}$ mice and control IFN- $\gamma^{\mathrm{CNS}-} ;$ Perk $^{+/+}$mice, around PID14, but the clinical severity was

\section{Figure 1}

CNS delivery of IFN- $\gamma$ before EAE onset ameliorated the disease course dependent on the PERK pathway. (A) Expression pattern of total IFN- $\gamma$ in the course of EAE $(n=3)$. (B) Expression pattern of transgenic IFN- $\gamma$ in the course of $\operatorname{EAE}(n=3)$. (C) Mean clinical score $(n=25)$. Error bars represent SD. ${ }^{*} P<0.05$, ${ }^{* *} P<0.01$ versus IFN- $\gamma^{\mathrm{CNS}-} ;$ Perk ${ }^{+/+}$; Student's $t$ test.

significantly milder in the IFN- $\gamma^{\mathrm{CNS}+} ; \mathrm{Perk}^{+/+}$mice (Figure 1C and Table 1). While all mice developed a typical EAE clinical phenotype, a significantly higher portion (20 of 25) of control IFN- $\gamma^{\mathrm{CNS}-} ; \mathrm{Perk}^{+/+}$ mice showed the severe EAE symptom of hind-limb paralysis compared with the IFN- $\gamma^{\mathrm{CNS}+} ; \mathrm{Perk}^{+/+}$mice ( 2 of 25 ; Table 1 ). Thus, these data confirm that CNS expression of IFN- $\gamma$ before EAE onset attenuates disease severity.

Spinal cord tissue was prepared at the peak of disease and analyzed for tissue damage. Control mice exhibited typical EAE histopathology: destruction of myelin sheaths, axon damage, and oligodendrocyte loss (Figure 2, A-E) as well as perivascular inflammation (Figure 3, A-D). In contrast, myelin, axons, and oligodendrocytes remained largely intact in the lumbar spinal cords of IFN- $\gamma^{\mathrm{CNS}+} ;$ Perk $^{+/+}$mice (Figure 2, A-E, and Supplemental Figure 1; supplemental material available online with this article; doi:10.1172/JCI29571DS1), and CD3-positive T cell infiltration was reduced (Figure 3, A, C, and D). Moreover, real-time PCR analyses revealed that the mRNA level of myelin basic protein (MBP)

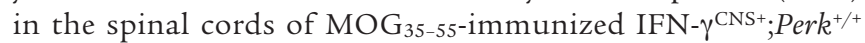
mice was not significantly decreased compared with age-matched nonimmunized, EAE-negative (i.e., healthy) mice. In contrast, the expression of MBP was significantly decreased in $\mathrm{MOG}_{35-55}$-immunized control IFN- $\gamma^{\mathrm{CNS}-} ;$ Perk $^{+/+}$mice, by approximately $30 \%$ and $22 \%$, compared with naive and IFN- $\gamma^{\mathrm{CNS}+} ;$ Perk $^{+/+}$mice, respectively (Figure 2F). These data indicate that preemptive CNS delivery of IFN- $\gamma$ protects against EAE-induced demyelination.

During EAE pathogenesis, $T$ cells are primed in the peripheral immune system and enter the CNS well before the onset of clinical disease (23). Preemptive derepression of the TRE/Ifng transgene to deliver the cytokine to the CNS before EAE onset did not affect $T$ cell priming in the periphery (Supplemental Figure 2). Accordingly, we found that CNS delivery of IFN- $\gamma$ did not significantly affect early infiltration by CD3-positive T cells at PID14 (Figure 3, E, F, I, and $\mathrm{J})$ and had no significant effect on the number of CD11b-positive microglia/macrophages in the spinal cord at this time point (Figure 3, G-J). Importantly, real-time PCR analyses showed that CNS delivery of IFN- $\gamma$ strongly enhanced the expression of iNOs, TNF- $\alpha$, IL-2, IL-12, and IL-10 in the spinal cord at EAE onset (Figure 3K); however, the derepression of the TRE/Ifng transgene did not signifi-
Table 1

PERK-dependent attenuated disease severity induced by CNS delivery of IFN- $\gamma$ before EAE onset

\begin{tabular}{|c|c|c|c|c|}
\hline Group & $\begin{array}{l}\text { Time of EAE } \\
\text { onset (PID) }\end{array}$ & $\begin{array}{l}\text { Clinical score at } \\
\text { disease peak }\end{array}$ & $\begin{array}{c}\text { Incidence of } \\
\text { hind-limb paralysis }\end{array}$ & $\begin{array}{l}\text { Incidence } \\
\text { of death }\end{array}$ \\
\hline IFN- $\gamma^{\text {CNS-}-; P e r k ~}{ }^{+/+}$ & $13.6 \pm 1.2$ & $2.66 \pm 0.80$ & $20 / 25$ & $0 / 25$ \\
\hline IFN- $\gamma^{\mathrm{CNS}+} ;$ Perk $^{+/+}$ & $14.7 \pm 1.4$ & $1.63 \pm 0.51^{A}$ & $2 / 25$ & $0 / 25$ \\
\hline IFN- $\gamma^{\text {CNS-; }}$ Perk ${ }^{+1-}$ & $14.0 \pm 0.9$ & $2.67 \pm 0.94$ & $19 / 25$ & $2 / 25$ \\
\hline IFN- $\gamma^{\mathrm{CNS}+} ;$ Perk ${ }^{+1-}$ & $13.6 \pm 0.8$ & $2.71 \pm 1.11$ & $18 / 25$ & $3 / 25$ \\
\hline
\end{tabular}

Data are mean $\pm \mathrm{SD}(n=25) .{ }^{\mathrm{A} P}<0.001$ versus IFN- ${ }^{\mathrm{CNS}-} ;$ Perk $^{+/+}$. 

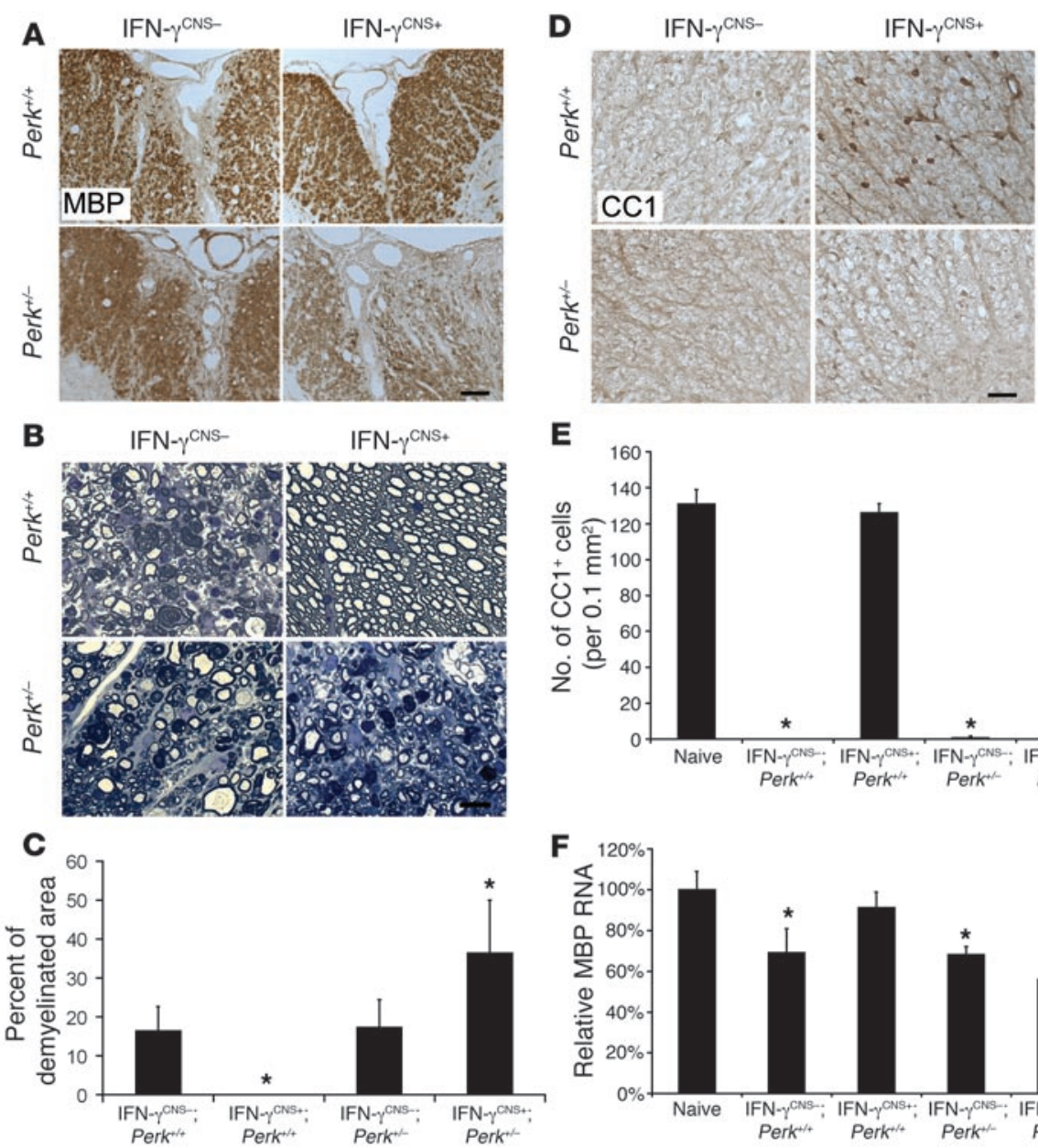

E
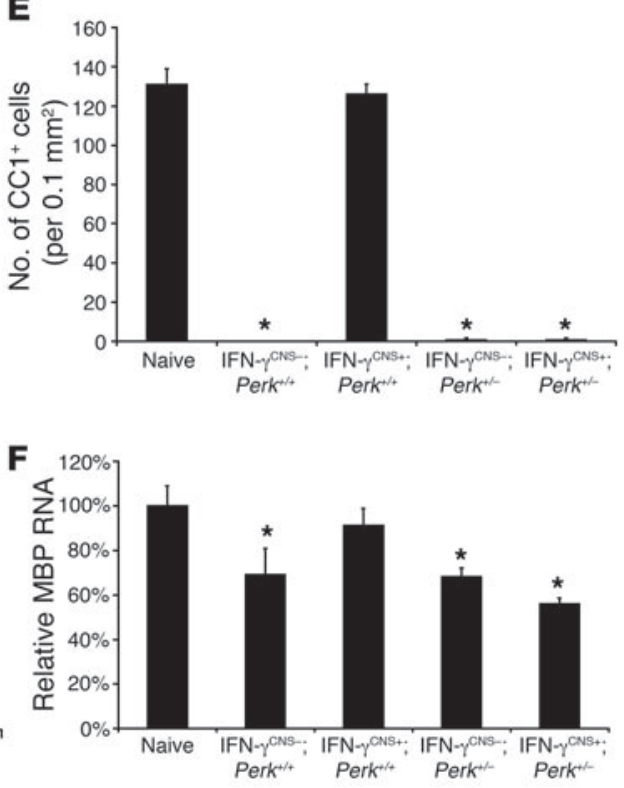

\section{Figure 2}

CNS delivery of IFN- $\gamma$ before EAE onset protects against EAE-induced demyelination dependent on the PERK pathway. (A) MBP immunostaining showed that the CNS delivery of IFN- $\gamma$ protected against EAE-induced demyelination in the lumbar spinal cords of mice on a Perk ${ }^{+/+}$background at PID17. In contrast, there was more severe demyelination in the lumbar spinal cord of IFN- $\gamma^{\mathrm{CNS}+} ; P_{\mathrm{erk}}^{+/-}$mice at PID17 compared with control mice. Scale bar: $50 \mu \mathrm{m}$. (B and C) Toluidine blue staining revealed that the myelin and axons in the spinal cords of IFN- $\gamma^{\mathrm{CNS}+;} ; \mathrm{Perk}^{+/+}$mice remained almost intact at PID17. In contrast, CNS delivery of IFN- $\gamma$ did not prevent demyelination and axon damage in the lumbar spinal cord of mice on a Perk ${ }^{+/}$ background at PID17. Scale bar: $10 \mu \mathrm{m}$. (D and E) CC1 immunostaining showed that oligodendrocytes in the lumbar spinal cords of IFN- $\gamma^{\mathrm{CNS}+} ; \mathrm{Perk}^{+/+}$mice remained almost intact at PID17. In contrast, similar to control mice, IFN- $\gamma^{\mathrm{CNS}+} ; \mathrm{Perk}^{+/-}$mice lost the majority of oligodendrocytes in the demyelinated lesions of their lumbar spinal cords at PID17. Scale bar: $25 \mu \mathrm{m}$. (F) Real-time PCR analysis of the mRNA level of MBP in the indicated spinal cords at PID17 relative to that in the spinal cords of age-matched naive mice. Error bars represent SD. $n=3$. ${ }^{\star} P<0.05$ versus IFN- $\gamma^{\mathrm{CNS}^{-} ; \mathrm{PERK}^{+/+}}$(C) or naive mice $(\mathbf{E}$ and $\mathbf{F})$. cantly affect the expression of iNOs or TNF- $\alpha$ at the peak of disease (Figure 4, A and B). We also found that CNS delivery of IFN- $\gamma$ did not change CD11b-positive microglia/macrophage numbers at the peak of disease (Figure 3, B-D). Moreover, enforced IFN- $\gamma$ expression did not significantly alter IL-17 mRNA levels at EAE onset or at the peak of disease (Figure $3 \mathrm{~K}$ and Figure $4 \mathrm{H}$ ). Taken together, these observations indicate that IFN- $\gamma$-mediated protection from EAE-induced demyelination is unlikely to be mediated by its previously documented antiinflammatory properties $(13,14)$.

The protective effects of IFN- $\gamma$ on EAE are associated with activation of the ISR in oligodendrocytes. There is accumulating evidence that oligodendrocyte death contributes significantly to the development of MS and $\operatorname{EAE}(4,5,24,25)$. Transgenic mice that specifically express antiapoptotic proteins in oligodendrocytes are resistant to EAE induction (26-28). We have previously reported that transgenic mice that express low levels of IFN- $\gamma$ in the CNS are resistant to toxicant-induced (i.e., with cuprizone) oligodendrocyte apoptosis and demyelination (29). Furthermore, we have demonstrated that IFN- $\gamma$ is capable of exerting direct effects on oligodendrocytes through the activation of the JAK/STAT signaling pathway (30). Taken together, these previous findings suggest that IFN- $\gamma$ protects against demyelination through a direct cytoprotective effect in oligodendrocytes.

We next sought to determine a molecular basis for the protective effects of IFN- $\gamma$. It is well established that low levels of cellular stress that activate downstream signaling pathways without causing severe cell injury can protect against subsequent exposure to more severe stresses $(3,31)$. Several lines of evidence have suggested that modest ER stress protects cells from TNF- $\alpha$ exposure, oxidative/nitrative injury, and glutamate excitoxicity $(1,3,32,33)$. Importantly, these 3 events are believed to play critical roles in oligodendrocyte death in MS and EAE $(4,34)$. We have previously shown that the presence of IFN- $\gamma$ in the CNS of adult mice induces modest ER stress, but does not cause myelin abnormalities or oligodendrocyte loss (20). The PERK-p-eIF2 $\alpha$ pathway balances protein biosynthesis with ER-folding capacity and contributes to the activation of most genes in the ER stress response $(1,3)$. It has been demonstrated that preemptive conditional activation of the PERK-p-eIF $2 \alpha$ pathway protects cells from the lethal effects of reactive oxidative/nitrative stress and ER stress (3). To determine whether the PERK pathway is involved in the protective effects of IFN- $\gamma$ in EAE-induced demyelination, we monitored the level of p-PERK and p-eIF $2 \alpha$ in oligodendrocytes during the course of EAE. Colocalization analysis with the CC1 antibody revealed that few oligodendrocytes were p-PERK-positive and p-eIF $2 \alpha$-positive in the spinal cords of control mice at EAE onset, consistent with a previous report (35). In contrast, CNS delivery of IFN- $\gamma$ markedly enhanced the activation of the PERK-eIF $2 \alpha$ pathway in oligodendrocytes of IFN- $\gamma^{\mathrm{CNS}+} ; \mathrm{Perk}^{+/+}$mice (Figure 5). Double immunolabeling analyses showed that the immunoreactivity of p-PERK was undetectable in other types of cells such as CD3-positive $\mathrm{T}$ cells, CD11b-positive microglial cells, and GFAP-positive astrocytic cells in the lumbar spinal cords of IFN- $\gamma^{\mathrm{CNS}+} ; \mathrm{Perk}^{+/+}$or 

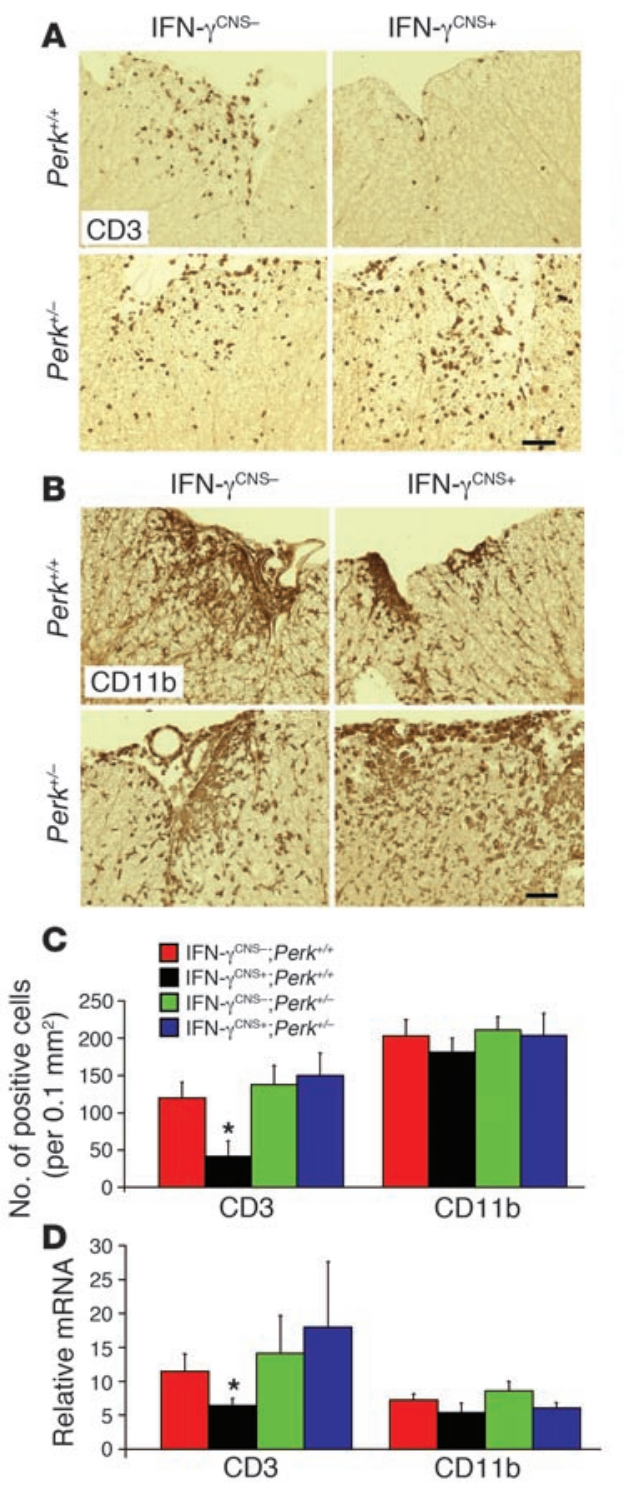
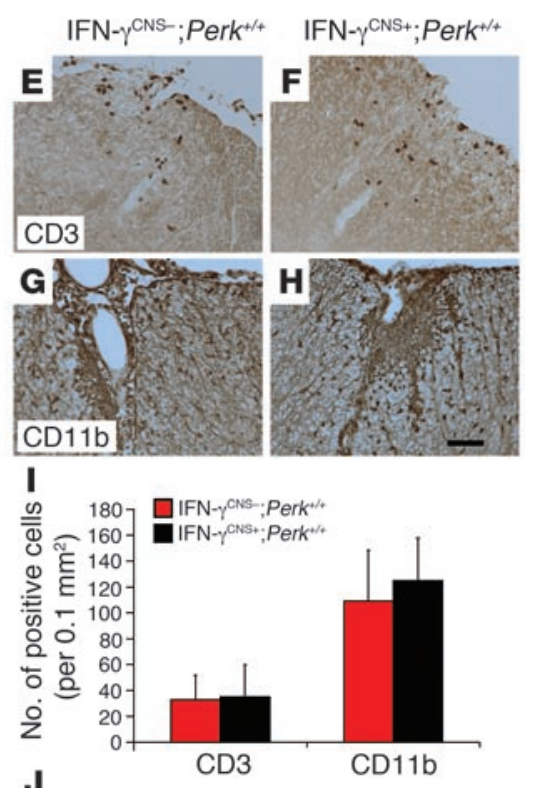

J
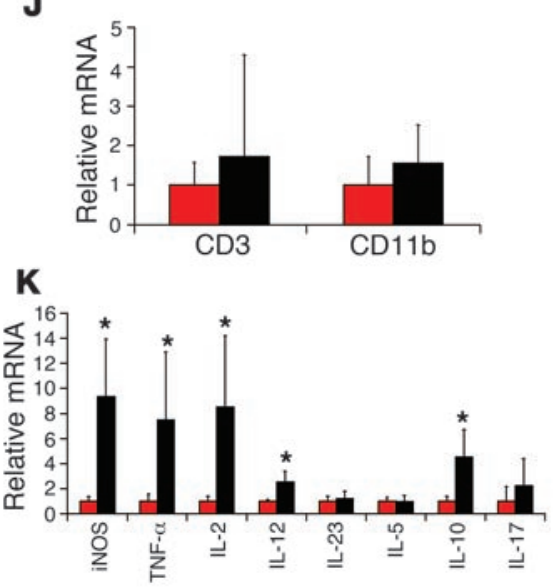

\section{Figure 3}

The effects of IFN- $\gamma$ on inflammatory infiltration. (A and $\mathbf{C}$ ) CD3 immunostaining showed that CNS delivery of IFN- $\gamma$ reduced $T$ cell infiltration in the lumbar spinal cords of mice on a Perk ${ }^{+/+}$background at PID17, but did not significantly affect $\mathrm{T}$ cell infiltration in mice on a Perk ${ }^{+/-}$background. (B and $\mathbf{C}$ ) CD11b immunostaining revealed that CNS delivery of IFN- $\gamma$ did not significantly change the numbers of CD11b-positive microglia/ macrophages in the lumbar spinal cord of mice on a Perk ${ }^{+/+}$or Perk ${ }^{+/}$background at PID17 ( $n=3)$. (D) Real-time PCR analysis of the relative mRNA levels of CD3 and CD11b in the spinal cord at PID17 $(n=3)$. (E, F, and I) CD3 immunostaining showed that CNS delivery of IFN- $\gamma$ did not affect $\mathrm{T}$ cell infiltration in lumbar spinal cord at PID14. (G, $\mathbf{H}$, and I) CD11b immunostaining showed that CNS delivery of IFN- $\gamma$ did not significantly change the numbers of CD11b-positive microglia/macrophages in the lumbar spinal cord at PID14 $(n=3)$. (J) Real-time PCR analysis of the relative mRNA levels of CD3 and CD11b in the spinal cord at PID14 $(n=4)$. (K) Real-time PCR analysis for the expression pattern of cytokines in the spinal cord at PID14 $(n=4)$. Scale bars: $50 \mu \mathrm{m}$. Error bars represent SD. ${ }^{*} P<0.05$ versus IFN- $\gamma^{\mathrm{CNS}-} ;$ Perk $^{+/+}$.
IFN- $\gamma^{\text {CNS- }} ;$ Perk $^{+/+}$mice at PID14 (Supplemental Figure 3). These data establish a correlation between the activation of the PERK-mediated ISR in oligodendrocytes and the protective role of IFN- $\gamma$ against EAE-induced demyelination.

PERK is essential for the protective effects of IFN- $\gamma$ in EAE. To explore a possible functional role of the ISR in protecting against EAE, we took a genetic approach. Mice that are heterozygous for a loss-of-function mutation in their Perk gene (also known as Eif2ak3) appear healthy but display evidence of haploid insufficiency $(20,21,36)$. We crossed TRE/Ifng mice with Perk ${ }^{+/-}$mice (37) and then crossed the resulting progeny with GFAP/tTA mice to obtain compound GFAP/tTA;TRE/Ifng;Perk ${ }^{+/-}$mice and control GFAP/tTA;TRE/Ifng;Perk ${ }^{+/+}$mice. Six-week-old GFAP/tTA;TRE/ Ifng;Perk ${ }^{+/-}$mice that had been maintained under doxycycline repression from conception were immunized with the $\mathrm{MOG}_{35-55}$ peptide and simultaneously released from doxycycline repression $\left(\mathrm{IFN}-\gamma^{\mathrm{CNS}+} ; \mathrm{Perk}^{+/-}\right)$. In marked contrast with $\mathrm{MOG}_{35-55}$-immunized IFN- $\gamma^{\mathrm{CNS}+} ; \mathrm{Perk}^{+/+}$mice, CNS delivery of IFN- $\gamma$ did not ameliorate the EAE disease course in IFN- $\gamma^{\mathrm{CNS}+} ;$ Perk $^{+/-}$mice (Figure $1 \mathrm{C}$ and
Table 1). Rather, the morbidity, disease severity, and mortality of

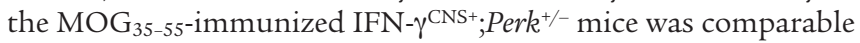
to that of control IFN- $\gamma^{\mathrm{CNS}-} ; \mathrm{Perk}^{+/-}$mice.

Neuropathologic analyses revealed that CNS delivery of IFN- $\gamma$ before EAE onset did not prevent demyelination, axon damage, or oligodendrocyte loss (Figure 2) and did not change the immune response at the peak of disease in the spinal cords of Perk ${ }^{+/-}$mice (Figure 3, A-D, and Figure 4). In fact, MBP immunostaining, toluidine blue staining, and real-time PCR analysis for MBP consistently showed comparable demyelination at the peak of disease in the spinal cords of IFN- $\gamma^{\mathrm{CNS}+} ; \mathrm{Perk}^{+/-}$and control IFN- $\gamma^{\mathrm{CNS}-} ; \mathrm{Perk}^{+/-}$ or IFN- $\gamma^{\mathrm{CNS}-} ; \mathrm{Perk}^{+/+}$mice (Figure 2). Importantly, the Perk ${ }^{+/}$genotype did not significantly affect $\mathrm{T}$ cell priming in the peripheral immune system (Supplemental Figure 2), inflammatory infiltration, or the expression profile of cytokines in the course of EAE (Figure 3, A-D, and Figure 4), indicating that PERK does not modulate the primary inflammatory response in EAE. Thus, the activation of the PERK pathway by IFN- $\gamma$ in oligodendrocytes is essential to the protection against EAE-induced demyelination. 
A

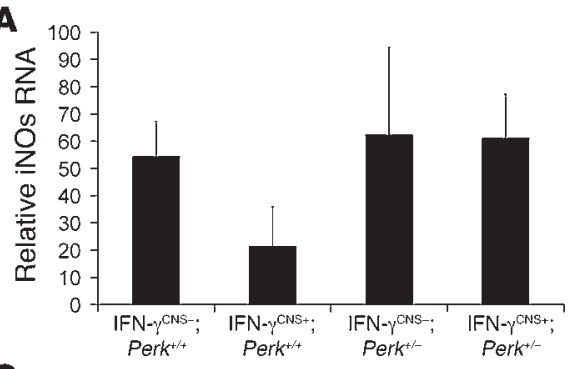

\section{C}

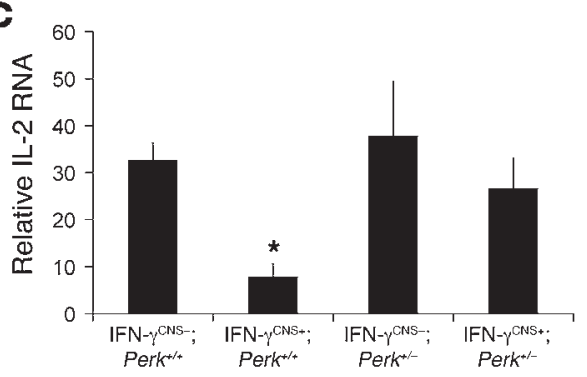

E

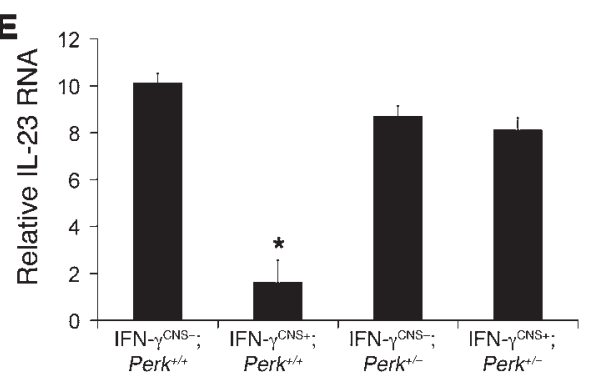

G

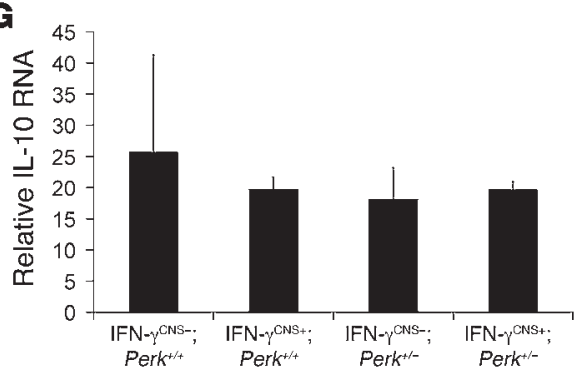

B

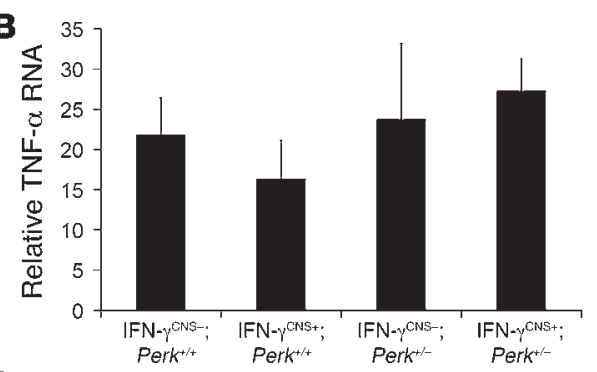

D

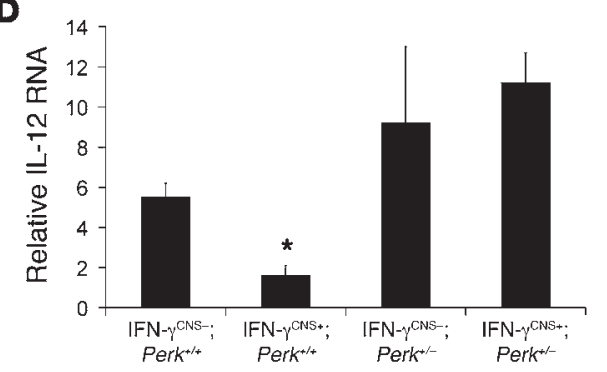

$\mathbf{F}$

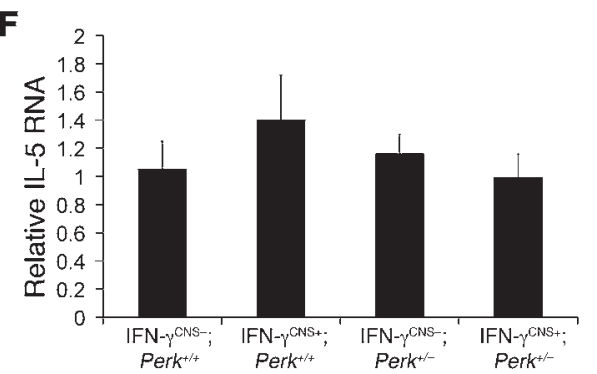

H

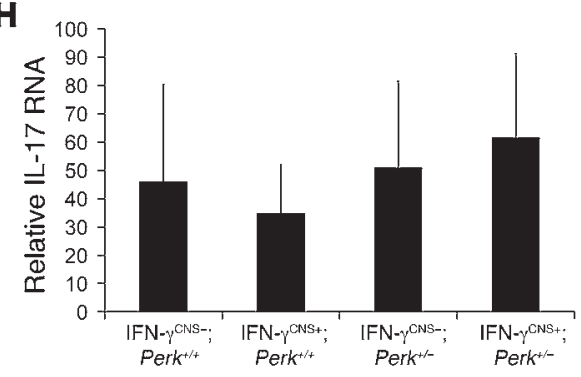

Figure 4

Real-time PCR analysis for the expression pattern of cytokines in the spinal cord at the peak of disease. (A) CNS delivery of IFN- $\gamma$ did not significantly affect the expression of iNOs. (B) CNS delivery of IFN- $\gamma$ did not significantly affect the expression of TNF- $\alpha$. (C) CNS delivery of IFN- $\gamma$ decreased the expression of IL-2 in the spinal cords of mice on a Perk ${ }^{+/+}$background, but did not change IL-2 expression in mice on a Perk'+- background. (D) CNS delivery of IFN- $\gamma$ decreased the expression of IL-12 in the spinal cords of mice on a Perk ${ }^{+/+}$background, but did not change IL-12 expression in mice on a Perk $^{+/-}$background. (E) CNS delivery of IFN- $\gamma$ decreased the expression of IL-23 in the spinal cords of mice on a Perk ${ }^{+/+}$ background, but did not change IL-23 expression in mice on a Perk ${ }^{+/}$background. (F) CNS delivery of IFN- $\gamma$ did not significantly affect the expression of IL-5. (G) CNS delivery of IFN- $\gamma$ did not significantly affect the expression of IL-10. (H) CNS delivery of IFN- $\gamma$ did not significantly affect the expression of IL-17. Error bars represent SD. $n=3 .{ }^{*} P<0.05$ versus IFN- $\gamma^{\text {CNS-}} ;$ Perk $^{+/+}$.

\section{Discussion}

ISR activation by the ER stress response protects cells against subsequent cellular stresses. The ER stress response also controls an apoptotic program to eliminate cells whose folding problems in the ER can not be resolved by the adaptive coordinated responses (38, 39). While it has been shown that the apoptotic program controlled by the ER stress response plays an important role in the pathogenesis of various diseases such as diabetes mellitus and neurodegenerative diseases $(40,41)$, several lines of evidence suggest that a modest ER stress response that does not cause severe cell injury can protect against subsequent exposure to more severe stresses $(32,33,38,39)$. A recent study also shows that the ER stress response protects the heart from ischemia and reperfusion injury (42).

We have previously found that severe ER stress induced by IFN- $\gamma$ in myelinating oligodendrocytes during development or in remyelinating oligodendrocytes in cuprizone-induced demyelinated lesions causes oligodendrocyte apoptosis and myelin abnormalities, and that PERK protects oligodendrocytes from these detrimental consequences of ER stress $(20,21)$. Nevertheless, the presence of comparable levels of IFN- $\gamma$ in the CNS of adult mice induces only modest ER stress and does not diminish mature oligodendrocyte survival, even in heterozygous Perk ${ }^{+-}$mice (20). This presents a striking contrast to the deleterious effect of the mutation on survival of IFN- $\gamma$-exposed oligodendrocytes engaged in developmental myelination or pathologic remyelination $(20,21)$. Rather, as described here, modest ER stress induced by IFN- $\gamma$ in mature oligodendrocytes of adult mice protects against EAE-induced demyelination, axonal damage, and oligodendrocyte loss.

Interestingly, the detrimental or beneficial effects of the ER stress response induced by IFN- $\gamma$ do not correlate with the amount of IFN- $\gamma$ present in the CNS. The ER stress response induced by a very low dose of IFN- $\gamma$ (approximately $20 \mathrm{pg} / \mathrm{mg}$ ) in the demyelinated lesions of cuprizone-treated mice, or a median dose (approximately $200 \mathrm{pg} / \mathrm{mg}$ ) in the CNS of young, developing mice results in remyelinating oligodendrocyte or developing myelinating oligodendrocyte death, respectively $(20,21)$. In contrast, the ER stress response induced by a high dose of IFN- $\gamma$, approximately $500 \mathrm{pg} / \mathrm{mg}$ in the spinal cords of adult mice, protects oligoden- 


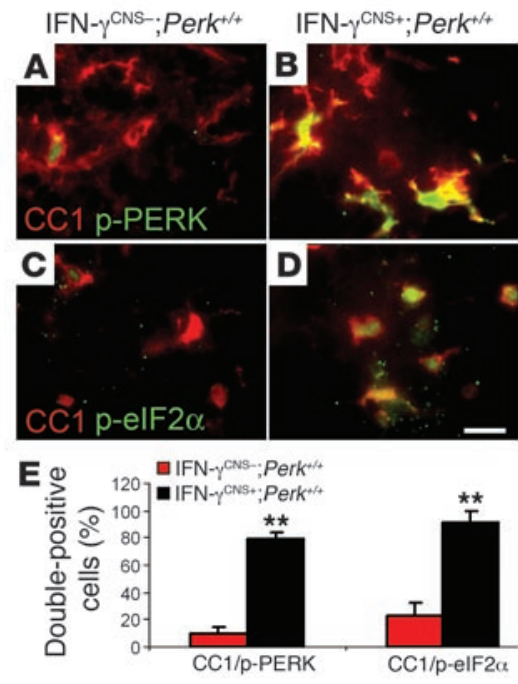

Figure 5

The protective effects of IFN- $\gamma$ in EAE are associated with activation of the PERK-elF2 $\alpha$ pathway in oligodendrocytes. (A and B) CC1 and p-PERK double labeling showed modest activation of PERK in a few oligodendrocytes in the lumbar spinal cords of control mice at PID14, and CNS delivery of IFN- $\gamma$ strongly activated PERK in the majority of oligodendrocytes. (C and D) CC1 and p-elF2 $\alpha$ double labeling showed that modest activation of elF2 $\alpha$ in a few oligodendrocytes in the lumbar spinal cord of control mice at PID14, and CNS delivery of IFN- $\gamma$ markedly activated elF2 $\alpha$ in the majority of oligodendrocytes. Scale bar: $10 \mu \mathrm{m}$. (E) Quantitative analysis showed CNS delivery of IFN- $\gamma$ significantly increased the percentage of double-positive CC1/p-PERK and $\mathrm{CC} 1 / \mathrm{p}$-elF2 $\alpha$ cells in the spinal cords of IFN- $\gamma^{\mathrm{CNS}+} ; \mathrm{Perk}^{+/+}$mice compared with IFN- $\gamma^{\mathrm{CNS}-;} \mathrm{Perk}^{+/+}$mice at PID14. Error bars represent SD. $n=3 .{ }^{* \star} P<0.01$ versus IFN- ${ }^{\mathrm{CNS}-} ; P \mathrm{Perk}^{+/+}$.

drocytes against immune-mediated damage. Thus, it is likely that the outcomes of ER stress induced by IFN- $\gamma$ in oligodendrocytes are determined by the developmental status of the cells. Oligodendrocytes produce a vast amount of myelin as an extension of their plasma membranes. During the active phase of myelination, each myelinating oligodendrocyte in the CNS must produce as much as approximately $5,000 \mu \mathrm{m}^{2}$ of myelin surface area per day and approximately $1 \times 10^{5}$ myelin protein molecules per minute (43). While the molecular mechanism by which IFN- $\gamma$ activates the ER stress response in oligodendrocytes is unclear, it is possible that IFN- $\gamma$ stresses the ER of oligodendrocytes by stimulating expression of hundreds of membrane-spanning proteins (particularly the antigen-presenting MHC molecules of the immune system) in these cells (K. Strand and B. Popko, unpublished observations). Previous studies have shown that the accumulation of MHC class I heavy chain molecules in the ER of oligodendrocytes results in myelin abnormalities in $M b p / H 2-K 1$ transgenic mice and that the expression of IFN- $\gamma$ in the CNS of these mice exacerbates that phenotype (44). Therefore, it is no great surprise that the ER stress response, of which the ISR is part, was unable to cope with the levels of ER stress in myelinating oligodendrocytes exposed to IFN- $\gamma$. Compared with actively myelinating oligodendrocytes in young, growing animals, mature oligodendrocytes in adult mice produce significantly lower levels of membrane proteins and lipids (45). ER homeostasis of mature oligodendrocytes in adult animals may thus be more easily maintained. Therefore, ER stress induced by IFN- $\gamma$ is less likely to trigger the apoptotic program in adult oligodendrocytes. Instead, ISR activation by modest ER stress may protect mature oligodendrocytes from immune-mediated insults. Importantly, our findings indicate that the apparently contradictory data previously reported regarding the role of IFN- $\gamma$ in MS and $\operatorname{EAE}(13,14)$ may be due at least in part to the biphasic effect of ER stress on oligodendrocyte survival.

eIF2 $\alpha$ phosphorylation protects cells from ER stress, oxidative/ nitrative injury, glutamate excitoxicity, and viral infection $(1-3,46)$. It is believed that phosphorylation of eIF $2 \alpha$ contributes to cytoprotection by blocking new proteins synthesis, which conserves energy, and by activating a transcriptional program that promotes the expression of numerous cytoprotective genes associated with the ISR $(1-3,38)$. Interestingly, the induction of ISR targeting genes such as that encoding activating transcription factor 4 (ATF4) has been demonstrated in MS lesions (47). We found modestly elevated p-eIF2 $\alpha$ and p-PERK immunoreactivity in oligodendrocytes in the course of EAE, as previously described (35). Moreover, CNS delivery of IFN- $\gamma$ before EAE onset markedly activated the PERK-eIF2 $\alpha$ pathway in oligodendrocytes, and the protective effects of IFN- $\gamma$ in EAE were dependent on the PERK pathway. Interestingly, 5 of the 50 mice that were heterozygous for the Perk mutation developed severe EAE and died around PID15 (Table 1), compared with the absence of mortality in Perk $^{+/+}$mice, further suggesting a protective function of PERK. Taken together, these data indicate that the PERK-eIF $2 \alpha$ pathway activated by the immune cytokine IFN- $\gamma$ in oligodendrocytes is necessary for cell survival and modulates the severity of disease in the pathogenesis of EAE.

An alternative, although not mutually exclusive, possibility is that CNS delivery of IFN- $\gamma$ before EAE onset protects against EAE-induced tissue damage through its immunomodulating functions. Furlan et al. reported that intrathecal delivery of IFN- $\gamma$ protected mice from EAE by increasing apoptosis of CNS-infiltrating T cells (18). In contrast, we did not observe increased apoptosis of infiltrating $\mathrm{T}$ cells in the CNS in our model (data not shown). We found that CNS delivery of IFN- $\gamma$ did not alter $\mathrm{T}$ cell infiltration at EAE onset, but did reduce $\mathrm{T}$ cell numbers at the peak of disease in the CNS of $\mathrm{Perk}^{+/+}$mice. Nevertheless, CNS delivery of IFN- $\gamma$ did not significantly affect $\mathrm{T}$ cell infiltration in the CNS of $\mathrm{Perk}^{+-}$mice. While a recent report suggests that phosphorylation of eIF $2 \alpha$ is involved in Th cell differentiation (48), our data (Supplemental Figure 2) show that CNS delivery of IFN- $\gamma$ did not significantly affect peripheral $\mathrm{T}$ cell responses. Importantly, the immunoreactivity of p-PERK was undetectable in CD3-positive T cells (Supplemental Figure 3), and the Perk mutation did not significantly affect $\mathrm{T}$ cell responses in the peripheral immune system or the CNS (Figures 3 and 4 and Supplemental Figure 2). In addition, a number of recent studies have reported that the inhibition of oligodendrocyte death reduces inflammatory infiltration in EAE lesions (26-28). Thus, it is possible that the protection that IFN- $\gamma$ provides against oligodendrocyte death results in the subsequent reduction of $T$ cell infiltration.

Recent studies have shown that IL-17 plays a critical role in the pathogenesis of $\operatorname{EAE}(49,50)$. It has also been demonstrated that IL-17 and IFN- $\gamma$ mutually suppress the development of IFN- $\gamma-$ and IL-17-producing effector T cells during an immune response $(49,50)$. Nevertheless, these cytokines cannot directly regulate the expression of each other in effector T cells (49). Interestingly, Komiyama et al. showed that the exacerbated EAE response in IFN- $\gamma$-deficient mice is associated with an increase in IL-17-pro- 
ducing $\mathrm{T}$ cells in the peripheral immune system (49), whereas Wheeler et al. reported that the elevated level of IFN- $\gamma$ in the CNS of TNF receptor 1-deficient mice protects against EAE but does not suppress the expression of IL-17 in the CNS (51). We show here that CNS delivery of IFN- $\gamma$ before EAE onset did not significantly affect the expression of IL-17 in the spinal cord at PID14 (Figure 3K) or PID17 (Figure 4H), whereas we have previously shown that CNS delivery of IFN- $\gamma$ at the recovery stage of EAE suppresses remyelination and significantly reduces the expression of IL-17 in the CNS (21). While an understanding of the effects of IFN- $\gamma$ on the production of IL-17 in the peripheral immune system and the CNS at different stages of EAE requires further investigation, these data collectively indicate that the harmful and helpful actions of IFN- $\gamma$ in EAE do not correlate with the level of IL-17 expression in the CNS.

Modulation of the microglia/macrophage response might also contribute to the observed effects of IFN- $\gamma$ on the pathogenesis of EAE. The inflammatory roles reported for microglia/macrophages in the pathogenesis of MS and EAE include the presentation of antigens and the production of inflammatory mediators such as TNF- $\alpha$ and iNOs (52). Butovsky et al. reported that microglia activated by low doses of IFN- $\gamma(20 \mathrm{ng} / \mathrm{ml})$ support oligodendrogenesis, but microglia activated by higher doses of IFN- $\gamma(100 \mathrm{ng} / \mathrm{ml})$ inhibit oligodendrogenesis (53). We found that CNS delivery of IFN- $\gamma$ did not significantly alter CD11b-positive microglia/macrophage infiltration or the induction of TNF- $\alpha$ and iNOs. MHC class II immunostaining also revealed that CNS delivery of IFN- $\gamma$ did not significantly change the infiltration of MHC class II-positive antigen-presenting cells (Supplemental Figure 4). Furthermore, our previous and current data demonstrate that the beneficial and detrimental effects of IFN- $\gamma$ on oligodendrocytes did not correlate with its dose $(20,21,29)$. It thus appears unlikely that suppressing inflammatory function of microglia/ macrophages contributes to the protective effects of high doses of IFN- $\gamma$ observed in our model.

Currently approved disease-modifying treatments for MS that nonspecifically target immune responses have little impact on the progression of disability $(54,55)$, which may in part reflect the dual roles, beneficial and detrimental, of many inflammatory components in this disease $(56,57)$. The results of the present study may have important implications for the pathogenesis of MS and could lead to therapeutic strategies to specifically protect oligodendrocytes in individuals with MS. Thus, the ability of the PERK response described here to protect against immunological insult on oligodendrocytes suggests that protective therapies that specifically activate the ISR may reduce relapse rate and delay disability progression in MS patients.

\section{Methods}

Mice and EAE immunization. Line110 of GFAP/tTA mice on the C57BL/6 background were mated with line $184 \mathrm{TRE} / \mathrm{Ifng}$ mice on the C57BL/6 background to produce GFAP/tTA;TRE/Ifng double transgenic mice (19). Moreover, TRE/Ifng mice were crossed with Perk ${ }^{+/}$mice on the C57BL/6 background (37), and the resulting progeny were crossed to GFAP/tTA mice to obtain GFAP/tTA;TRE/Ifng double transgenic mice that were heterozygous for the Perk mutation. To prevent transcriptional activation of the TRE/Ifng transgene by the tTA, $0.05 \mathrm{mg} / \mathrm{ml}$ doxycycline was added to the drinking water and provided ad libitum from conception. EAE was induced as described previously (21) by subcutaneous injections at flanks and tail base of $200 \mu \mathrm{g} \mathrm{MOG}_{35-55}$ emulsified in complete Freund's adjuvant
(BD) supplemented with $600 \mu \mathrm{g}$ Mycobacterium tuberculosis (strain H37Ra; $\mathrm{BD}$ ). Two intraperitoneal injections of $400 \mathrm{ng}$ pertussis toxin (List Biological Laboratories Inc.) were given 24 and 72 hours later. Clinical scores were recorded daily using a scale of $0-5$ ( 0 , healthy; 1 , flaccid tail; 2 , ataxia and/or paresis of hind limbs; 3 , paralysis of hind limbs and/or paresis of fore limbs; 4 , tetraparalysis; 5 , moribund or death).

All animal procedures were conducted in complete compliance with the NIH Guide for the Care and Use of Laboratory Animals and were approved by the Institutional Animal Care and Use Committee of The University of Chicago.

$T$ cell proliferation assay, cytokine assay, and ELISPOT assay. Single-spleen cell suspensions were generated from mice at PID10. Red blood cells were lysed with buffered Tris ammonium chloride, washed, and plated at $5 \times 10^{5}$ splenocytes/well in serum-free media. Samples were plated in triplicate. $\mathrm{OVA}_{323-339}$ or $\mathrm{MOG}_{35-55}$ was added at various concentrations as indicated in Supplemental Figure 2. The cells were incubated at $37^{\circ} \mathrm{C}$ and $7.5 \% \mathrm{CO}_{2}$. After 72 hours, cultures were pulsed with $\left[{ }^{3} \mathrm{H}\right]$-thymidine (1 mCi/well; MP Biomedicals) and harvested 16 hours later. We determined cpm with a TopCount-NXT microplate scintillation and luminescence counter (Packard). We quantified cytokines from culture supernatants of triplicate wells using the 5 plex cytokine bead array kit (QIAGEN) according to the manufacturer's instructions. ELISPOT assays were performed as previously described (58). We plated $2.5 \times 10^{5}$ splenocytes/well.

Real-time PCR. Anesthetized mice were perfused with PBS. RNA was isolated from the spinal cord using TRIzOL reagent (Invitrogen) and treated with DNAse I (Invitrogen) to eliminate genomic DNA. Reverse transcription was performed using the SuperScript First Strand Synthesis System for RT-PCR kit (Invitrogen). TaqMan real-time PCR was performed with iQ Supermix (Bio-Rad) on a Bio-Rad iQ Real-Time PCR detection system as previously described (21).

ELISA. Mice were perfused with ice-cold PBS. The spinal cords from 3 mice were removed, pooled, and immediately homogenized in 5 volumes of PBS with complete protease cocktail (Roche Diagnostics) using a motorized homogenizer. After incubation on ice for 5 minutes, the extracts were cleared by centrifugation at $10,000 \mathrm{~g}$ for 10 minutes. The protein content of each extract was determined by the DC protein assay (Bio-Rad). ELISA assays were performed using the Mouse IFN- $\gamma$ Quantikine ELISA kit (R\&D Systems) following the manufacturer's instructions.

Immunohistochemistry. Anesthetized mice were perfused with $4 \%$ paraformaldehyde in 0.1 M PBS. Immunohistochemistry for CC1 (adenomatus polyposis coli [antibody 7], diluted 1:50; EMD Biosciences), MBP (diluted 1:1,000; Sternberger Monoclonals Inc.), p-eIF2 $\alpha$ (diluted 1:50; Cell Signaling Technology), p-PERK (diluted 1:50; Cell Signaling Technology), CD3 (diluted 1:50; Santa Cruz Biotechnology Inc.), CD11b (diluted 1:50; Chemicon International), GFAP (diluted 1:1,000; Sternberger Monoclonals Inc.), and MHC class II (diluted 1:50; Chemicon International) was performed as previously described (20). We quantified the immunopositive cells by counting positive cells within the anteromedian white matter of the lumbar spinal cord, confined to an area of $0.1 \mathrm{~mm}^{2}$. The percentage of double-positive cells was calculated by normalizing CC1/p-PERK or CC1/p-eIF2 $\alpha$ double-positive cell numbers against CC1-positive cell numbers. The histological analysis was performed by a researcher blinded to experimental group.

Toluidine blue staining. For toluidine blue staining of $1-\mu \mathrm{m}$ sections, we anesthetized and perfused mice with $0.1 \mathrm{M}$ PBS containing $4 \%$ paraformaldehyde and $2.5 \%$ glutaraldehyde. The lumbar spinal cord were processed, embedded, sectioned, and analyzed as previously described (21). The total white matter area and the demyelinated area in the lumbar spinal cord were measured by ImageJ software (http://rsb.info.nih.gov/ij/). The percentage of demyelinated area was calculated by normalizing the demy- 
elinated area against the total white matter area. The histological analysis was performed by a researcher blinded to experimental group.

Statistics. Data are expressed as mean \pm SD. Multiple comparisons were statistically evaluated by 1-way ANOVA followed by the Holm-Sidak test using SigmaStat 3.1 software (Hearne Scientific Software). A $P$ value less than 0.05 was considered significant.

\section{Acknowledgments}

This work was supported by NIH grant NS34939 and the National Multiple Sclerosis Society (RG 3291 A4/T) to B. Popko, by the Myelin Repair Foundation to B. Popko and S.D. Miller, and by
NIH grants DK47119 and ES08681 to D. Ron. S.L. Bailey is supported by an NMSS Postdoctoral Fellowship Grant.

Received for publication July 5, 2006, and accepted in revised form November 14, 2006.

Address correspondence to: Brian Popko, Jack Miller Center for Peripheral Neuropathy, Department of Neurology, The University of Chicago, 5841 South Maryland Avenue MC2030, Chicago, Illinois 60637, USA. Phone: (773) 702-4953; Fax: (773) 702-5577; E-mail: bpopko@uchicago.edu.
1. Harding, H.P., et al. 2003. An integrated stress response regulates amino acid metabolism and resistance to oxidative stress. Mol. Cell. 11:619-633.

2. Proud, C.G. 2005. eIF2 and the control of cell physiology. Semin. Cell Dev. Biol. 16:3-12.

3. Lu, P.D., et al. 2004. Cytoprotection by pre-emptive conditional phosphorylation of translation initiation factor 2. EMBO J. 23:169-179.

4. Buntinx, M., Stinissen, P., Steels, P., Ameloot, M., and Raus, J. 2002. Immune-mediated oligodendrocyte injury in multiple sclerosis: molecular mechanisms and therapeutic interventions. Crit. Rev. Immunol. 22:391-424.

5. Frohman, E.M., Racke, M.K., and Raine, C.S. 2006. Multiple sclerosis-the plaque and its pathogenesis. N. Engl. J. Med. 354:942-955.

6. Popko, B., Corbin, J.G., Baerwald, K.D., Dupree, J., and Garcia, A.M. 1997. The effects of interferongamma on the central nervous system. Mol. Neurobiol. 14:19-35.

7. Imitola, J., Chitnis, T., and Khoury, S.J. 2005. Cytokines in multiple sclerosis: from bench to bedside. Pharmacol. Ther. 106:163-177.

8. Panitch, H.S., Hirsch, R.L., Schindler, J., and Johnson, K.P. 1987. Treatment of multiple sclerosis with gamma interferon: exacerbations associated with activation of the immune system. Neurology. 37:1097-1102.

9. Renno, T., et al. 1998. Interferon-gamma in progression to chronic demyelination and neurological deficit following acute EAE. Mol. Cell. Neurosci. 12:376-389.

10. Sun, D., Newman, T.A., Perry, V.H., and Weller, R.O. 2004. Cytokine-induced enhancement of autoimmune inflammation in the brain and spinal cord: implications for multiple sclerosis. Neuropathol. Appl. Neurobiol. 30:374-384.

11. Corbin, J.G., et al. 1996. Targeted CNS expression of interferon-gamma in transgenic mice leads to hypomyelination, reactive gliosis, and abnormal cerebellar development. Mol. Cell. Neurosci. 7:354-370.

12. LaFerla, F.M., Sugarman, M.C., Lane, T.E., and Leissring, M.A. 2000. Regional hypomyelination and dysplasia in transgenic mice with astrocytedirected expression of interferon-gamma. J. Mol. Neurosci. 15:45-59.

13. Muhl, H., and Pfeilschifter, J. 2003. Anti-inflammatory properties of pro-inflammatory interferongamma. Int. Immunopharmacol. 3:1247-1255.

14. Wheeler, R.D., and Owens, T. 2005. The changing face of cytokines in the brain: perspectives from EAE. Curr. Pharm. Des. 11:1031-1037.

15. Ferber, I.A., et al. 1996. Mice with a disrupted IFN-gamma gene are susceptible to the induction of experimental autoimmune encephalomyelitis (EAE). J. Immunol. 156:5-7.

16. Willenborg, D.O., Fordham, S., Bernard, C.C., Cowden, W.B., and Ramshaw, I.A. 1996. IFNgamma plays a critical down-regulatory role in the induction and effector phase of myelin oligodendrocyte glycoprotein-induced autoimmune encephalomyelitis. J. Immunol. 157:3223-3227.

17. Krakowski, M., and Owens, T. 1996. Interferon- gamma confers resistance to experimental allergic encephalomyelitis. Eur. J. Immunol. 26:1641-1646.

18. Furlan, R., et al. 2001. Intrathecal delivery of IFNgamma protects $\mathrm{C} 57 \mathrm{BL} / 6$ mice from chronicprogressive experimental autoimmune encephalomyelitis by increasing apoptosis of central nervous system-infiltrating lymphocytes. J. Immunol. 167:1821-1829.

19. Lin, W., et al. 2004. Interferon-gamma induced medulloblastoma in the developing cerebellum. J. Neurosci. 24:10074-10083.

20. Lin, W., Harding, H.P., Ron, D., and Popko, B. 2005. Endoplasmic reticulum stress modulates the response of myelinating oligodendrocytes to the immune cytokine interferon-gamma. J. Cell Biol. 169:603-612.

21. Lin, W., et al. 2006. Interferon-gamma inhibits central nervous system remyelination through a process modulated by endoplasmic reticulum stress. Brain. 129:1306-1318.

22. Okuda, Y., Sakoda, S., Bernard, C.C., and Yanagihara, T. 1998. The development of autoimmune encephalomyelitis provoked by myelin oligodendrocyte glycoprotein is associated with an upregulation of both proinflammatory and immunoregulatory cytokines in the central nervous system. J. Interferon Cytokine Res. 18:415-421.

23. Hickey, W.F., Hsu, B.L., and Kimura, H. 1991. Tlymphocyte entry into the central nervous system. J. Neurosci. Res. 28:254-260.

24. Hisahara, S., Okano, H., and Miura, M. 2003. Caspase-mediated oligodendrocyte cell death in the pathogenesis of autoimmune demyelination. Neurosci. Res. 46:387-397.

25. Matute, C., and Perez-Cerda, F. 2005. Multiple sclerosis: novel perspectives on newly forming lesions. Trends Neurosci. 28:173-175.

26. Hisahara, S., et al. 2000. Targeted expression of baculovirus p35 caspase inhibitor in oligodendrocytes protects mice against autoimmune-mediated demyelination. EMBO J. 19:341-348.

27. Hisahara, S., Yuan, J., Momoi, T., Okano, H., and Miura, M. 2001. Caspase-11 mediates oligodendrocyte cell death and pathogenesis of autoimmunemediated demyelination. J. Exp. Med. 193:111-122.

28. Hovelmeyer, N., et al. 2005. Apoptosis of oligodendrocytes via Fas and TNF-R1 is a key event in the induction of experimental autoimmune encephalomyelitis. J. Immunol. 175:5875-5884.

29. Gao, X., et al. 2000. Interferon-gamma protects against cuprizone-induced demyelination. Mol. Cell. Neurosci. 16:338-349.

30. Balabanov, R., Strand, K., Kemper, A., Lee, J.Y., and Popko, B. 2006. Suppressor of cytokine signaling 1 expression protects oligodendrocytes from the deleterious effects of interferon-gamma.J. Neurosci. 26:5143-5152.

31. Welch, W.J. 1992. Mammalian stress response: cell physiology, structure/function of stress proteins, and implications for medicine and disease. Physiol. Rev. 72:1063-1081.

32. Hung, C.C., Ichimura, T., Stevens, J.L., and Bonventre, J.V. 2003. Protection of renal epithelial cells against oxidative injury by endoplasmic reticulum stress preconditioning is mediated by ERK $1 / 2$ activation. J. Biol. Chem. 278:29317-29326.

33. Xue, X., et al. 2005. Tumor necrosis factor alpha (TNFalpha) induces the unfolded protein response (UPR) in a reactive oxygen species (ROS)-dependent fashion, and the UPR counteracts ROS accumulation by TNFalpha. J. Biol. Chem. 280:33917-33925.

34. Trapp, B.D., Bo, L., Mork, S., and Chang, A. 1999. Pathogenesis of tissue injury in MS lesions. J. Neuroimmunol. 98:49-56.

35. Chakrabarty, A., Danley, M.M., and LeVine, S.M. 2004. Immunohistochemical localization of phosphorylated protein kinase R and phosphorylated eukaryotic initiation factor- 2 alpha in the central nervous system of SJL mice with experimental allergic encephalomyelitis. J. Neurosci. Res. 76:822-833.

36. Harding, H.P., Zhang, Y., Bertolotti, A., Zeng, H., and Ron, D. 2000. Perk is essential for translational regulation and cell survival during the unfolded protein response. Mol. Cell. 5:897-904.

37. Harding, H.P., et al. 2001. Diabetes mellitus and exocrine pancreatic dysfunction in perk-/- mice reveals a role for translational control in secretory cell survival. Mol. Cell. 7:1153-1163.

38. Boyce, M., and Yuan, J. 2006. Cellular response to endoplasmic reticulum stress: a matter of life or death. Cell Death Differ. 13:363-373.

39. Schroder, M., and Kaufman, R.J. 2006. Divergent roles of IRE1alpha and PERK in the unfolded protein response. Curr. Mol. Med. 6:5-36.

40. Araki, E., Oyadomari, S., and Mori, M. 2003. Endoplasmic reticulum stress and diabetes mellitus. Intern. Med. 42:7-14.

41. Lindholm, D., Wootz, H., and Korhonen, L. 2006. ER stress and neurodegenerative diseases. Cell Death Differ. 13:385-392.

42. Martindale, J.J., et al. 2006. Endoplasmic reticulum stress gene induction and protection from ischemia/reperfusion injury in the hearts of transgenic mice with a tamoxifen-regulated form of ATF6. Circ. Res. 98:1186-1193.

43. Pfeiffer, S.E., Warrington, A.E., and Bansal, R. 1993. The oligodendrocyte and its many cellular processes. Trends Cell Biol. 3:191-197.

44. Baerwald, K.D., Corbin, J.G., and Popko, B. 2000. Major histocompatibility complex heavy chain accumulation in the endoplasmic reticulum of oligodendrocytes results in myelin abnormalities. J. Neurosci. Res. 59:160-169.

45. Morell, P., and Quarles, R.H. 1999. Myelin formation, structure and biochemistry. In Basic neurochemistry: molecular, cellular, and medical aspects. G.J. Siegel, B.W. Agranoff, R.W. Albers, S.K. Fisher, and M.D. Uhler, editors. Lippincott-Raven Publishers. Philadelphia, Pennsylvania, USA. 69-93.

46. Boyce, M., et al. 2005. A selective inhibitor of eIF2alpha dephosphorylation protects cells from ER stress. Science. 307:935-939.

47. Mycko, M.P., Papoian, R., Boschert, U., Raine, C.S., and Selmaj, K.W. 2004. Microarray gene expression profiling of chronic active and inactive lesions in multiple sclerosis. Clin. Neurol. 
Neurosurg. 106:223-229.

48. Scheu, S., et al. 2006. Activation of the integrated stress response during $\mathrm{T}$ helper cell differentiation. Nat. Immunol. 7:644-651.

49. Komiyama, Y., et al. 2006. IL-17 plays an important role in the development of experimental autoimmune encephalomyelitis. J. Immunol. 177:566-573.

50. Park, H., et al. 2005. A distinct lineage of CD4 T cells regulates tissue inflammation by producing interleukin 17. Nat. Immunol. 6:1133-1141.

51. Wheeler, R.D., Zehntner, S.P., Kelly, L.M., Bourbonniere, L., and Owens, T. 2006. Elevated interferon gamma expression in the central nervous system of tumour necrosis factor receptor 1-deficient mice with experimental autoimmune encephalomyelitis. Immunology. 118:527-538.

52. Raivich, G., and Banati, R. 2004. Brain microglia and blood-derived macrophages: molecular profiles and functional roles in multiple sclerosis and animal models of autoimmune demyelinating disease. Brain Res. Brain Res. Rev. 46:261-281.

53. Butovsky, O., et al. 2006. Induction and blockage of oligodendrogenesis by differently activated microglia in an animal model of multiple sclerosis. . Clin. Invest. 116:905-915. doi:10.1172/JCI26836.

54. Killestein, J., and Polman, C.H. 2005. Current tri- als in multiple sclerosis: established evidence and future hopes. Curr. Opin. Neurol. 18:253-260.

55. Kapoor, R. 2006. Neuroprotection in multiple sclerosis: therapeutic strategies and clinical trial design. Curr. Opin. Neurol. 19:255-259.

56. Franklin, R.J. 2002. Why does remyelination fail in multiple sclerosis? Nat. Rev. Neurosci. 3:705-714.

57. Ransohoff, R.M., and Trapp, B.D. 2005. Taking two TRAILS. Neuron. 46:355-356.

58. Tompkins, S.M., et al. 2002. De novo central nervous system processing of myelin antigen is required for the initiation of experimental autoimmune encephalomyelitis. J. Immunol. 168:4173-4183. 